\title{
First Light with the HAWC Gamma-Ray Observatory
}

\author{
Stefan Westerhoff*, for the HAWC Collaboration \\ Wisconsin IceCube Particle Astrophysics Center (WIPAC) and Department of Physics, \\ University of Wisconsin-Madison, Madison, WI 53706, USA \\ E-mail: westerhoff@wisc.edu
}

The High-Altitude Water Cherenkov Gamma-Ray Observatory (HAWC) is currently under construction 4,100 meters above sea level on the slope of Pico de Orizaba, Mexico. HAWC is a large field-of-view instrument capable of continuously monitoring the gamma-ray sky between roughly $50 \mathrm{GeV}$ and $100 \mathrm{TeV}$. The detector will be used to record both steady and transient gamma-ray sources and to provide an unbiased survey of the northern sky. Upon completion, HAWC will comprise 300 large light-tight water tanks covering an area of 20,000 square meters. Each tank will be instrumented with four photomultipliers to detect particles from extensive air showers produced by gamma rays and cosmic rays. Since summer 2013, more than 100 tanks have been operating in data acquisition mode. Event statistics and sensitivity are already sufficient to perform studies of cosmic rays and flaring gamma-ray sources. We report results from the first months of detector operation, including the detection of the cosmic ray shadow of the Moon and small-scale anisotropy in the arrival direction distribution of cosmic rays.

The European Physical Society Conference on High Energy Physics -EPS-HEP2013

18-24 July 2013

Stockholm, Sweden

\footnotetext{
${ }^{*}$ Speaker.
} 


\section{The HAWC Observatory}

The High-Altitude Water Cherenkov (HAWC) Observatory near Pico de Orizaba in Mexico $\left(\mathrm{N} 18^{\circ} 59^{\prime}, \mathrm{W} 97^{\circ} 18^{\prime}\right)$ is a detector designed to observe gamma rays and cosmic rays in the energy range from about $50 \mathrm{GeV}$ to $100 \mathrm{TeV}$. The study of these high-energy particles provides us with a better understanding of some of the most extreme astrophysical objects, from supernova remnants in our Galaxy to extragalactic objects like active galactic nuclei (AGN) and gamma-ray bursts (GRBs).

High-energy gamma-ray emission correlates with sites of cosmic-ray acceleration, so the observation of the sky in high-energy gamma rays is a promising approach to understanding the origin of Galactic and extragalactic cosmic rays. Charged cosmic rays cannot be expected to point back to their sources except at the highest energies (some $10^{19} \mathrm{eV}$ and above) because of deflection in magnetic fields. Gamma rays, however, do point back to their sources. By locating and studying high-energy gamma-ray sources, we ultimately hope to understand where and how cosmic rays are accelerated.

At $\mathrm{TeV}$ energies, the atmosphere is opaque to gamma rays. On entering the Earth's atmosphere, the gamma rays interact with air molecules and induce large cascades of secondary particles, so-called air showers. The properties of the gamma-ray primary have to be reconstructed from the air-shower cascade it induces.

Upon completion, the HAWC detector will comprise a large $\left(150 \times 150 \mathrm{~m}^{2}\right)$ array of 300 light-tight water tanks, each 4.7 meters high and 7.3 meters in diameter. Fig. 1 (left) shows the planned layout of the detector. Each water tank will be instrumented with four photomultiplier tubes (PMTs), which record the Cherenkov light produced when the particles of the extensive air shower reach the ground and traverse the detector (Fig. 1 (right)). Since the air-shower particles arrive nearly in a plane, the relative time of light arriving in the PMTs can be used to determine the direction of the particle that initiated the shower. HAWC can be operated in all weather conditions and ambient light levels and therefore has a theoretical duty cycle close to $100 \%$. It has a large
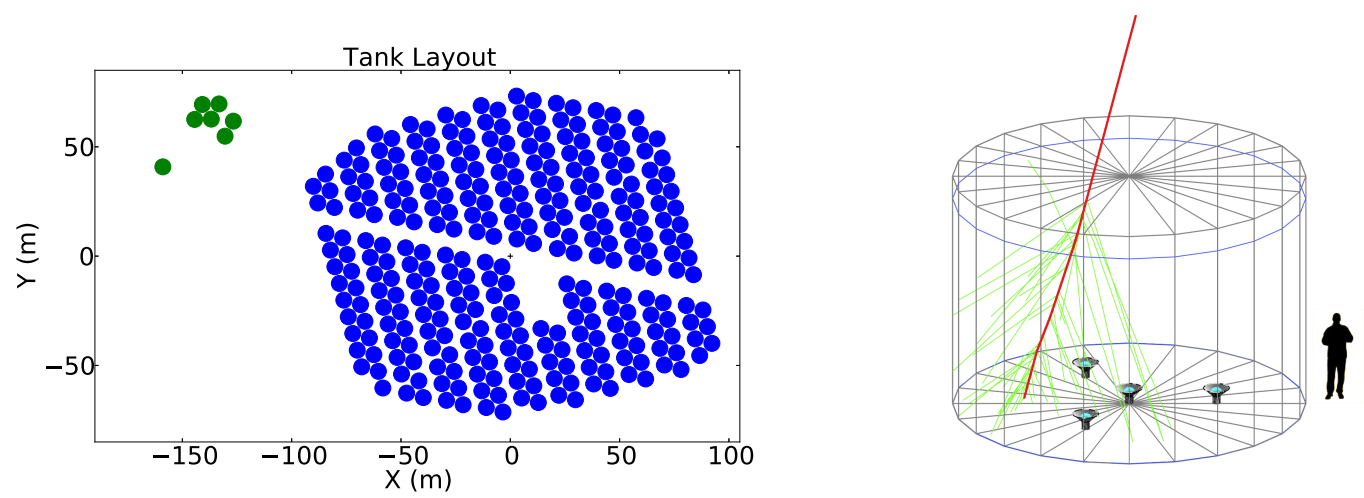

Figure 1: Left: Layout of the full HAWC detector with 300 tanks. Each blue circle indicates the position of a HAWC tank. The green dots on the upper left indicate the location of the VAMOS array, a small test array operated in 2011. The gap in the middle of the HAWC array marks the location of the central electronics building. Right: Schematic of a HAWC tank. 


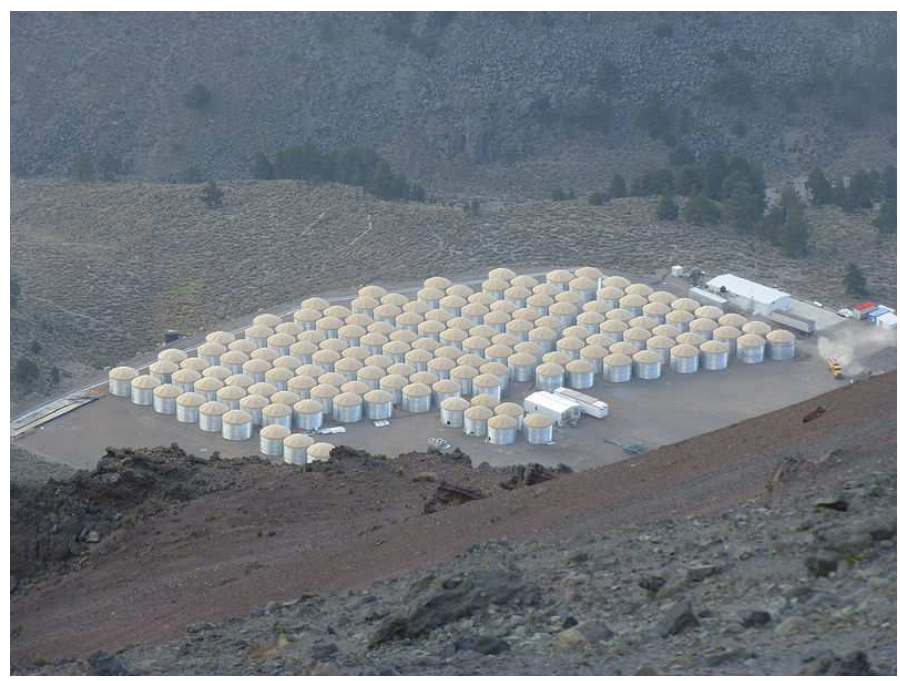

Figure 2: Recent photograph of the HAWC site (Fall 2013), with more than 140 completed tanks.

effective area and an instantaneous field of view of about 2 sr (or $16 \%$ of $4 \pi$ ), with a daily sky coverage of $2 \pi \mathrm{sr}$. Simulations show that the angular resolution of HAWC is about $0.1^{\circ}$ for energies greater than $10 \mathrm{TeV}$. The energy resolution is around $30 \%$ at energies above $10 \mathrm{TeV}$.

Ground-based instruments like HAWC have to detect gamma-ray sources against the background of charged cosmic rays, which are more than 1,000 times more abundant than gamma rays at $\mathrm{TeV}$ energies. Separation of gamma-induced showers from hadron-induced showers is therefore one of the most crucial tasks of data analysis in gamma-ray astronomy. In HAWC, the analysis of the two-dimensional pattern of triggered PMTs and their charge provides a means to separate hadron- and gamma-induced showers. The lateral profile of gamma showers is relatively smooth due to their almost purely electromagnetic nature. Hadron-induced showers have a messy profile with "hot spots" from penetrating particles far from the shower core. By tagging all events with a large amount of charge far away from the core as hadronic, it is possible to reject more than $99 \%$ of the background for events above $10 \mathrm{TeV}$. The remaining cosmic-ray background is irreducible and needs to be estimated to identify gamma-ray sources (see, e.g., [1] for a description of the background estimation method).

As of summer 2013, more than 100 tanks have been built and instrumented, and data-taking in this configuration has started. Fig. 2 shows a recent photograph of the detector. The full detector is expected to be completed in late 2014 .

\section{HAWC Science Goals}

HAWC is unique among gamma-ray detectors in that it provides a continuous unbiased survey of the TeV gamma sky. Unlike imaging Cherenkov telescopes, HAWC has a field of view of about two-thirds of the sky and will be sensitive to extended sources.

The science topics that can be addressed with HAWC range from gamma-ray astronomy and direct studies of $\mathrm{TeV}$ cosmic rays to a variety of topics in astrophysics and particle physics. Here, 
we list some of the most important science goals of HAWC.

- First and foremost, HAWC will provide an unbiased map of the TeV sky with a daily coverage of $2 \pi$ sr. It will search for transient phenomena like GRBs and flares in AGN, and contribute to multiwavelength campaigns that study a selected object at various wavelengths, from radio to $\mathrm{TeV}$ gamma rays, at the same time. It will be able to provide $\mathrm{TeV}$ alerts for other instruments, for example, imaging air Cherenkov telescopes, that can follow up with detailed observations of any flare region discovered by HAWC. The sensitivity of HAWC to steady sources of $\mathrm{TeV}$ gamma-ray emission is described in detail in [2], and the sensitivity to GRBs is described in [3].

- HAWC will provide important clues on the origin of Galactic cosmic rays. It is generally believed that supernova explosions are responsible for the cosmic-ray flux up to about $10^{15} \mathrm{eV}$. There is currently no direct evidence for this scenario, but we know that the power released in supernovae is sufficient to sustain the Galactic cosmic-ray flux. An important tool in understanding the origin of cosmic rays is the observation of high-energy gamma rays from localized sources. Gamma rays can be generated when accelerated cosmic rays interact in or near their source. However, the observation of gamma rays is not necessarily a "smoking gun" for cosmic-ray acceleration, because high-energy gamma rays can also be produced by inverse-Compton scattering of low energy photons to high energies by ultrarelativistic electrons. Potential cosmic-ray accelerators can be identified by measuring the energy spectra of sources. A typical electron-produced gamma-ray apectrum would cut off earlier than a typical cosmic-ray-produced spectrum because of Klein-Nishina effects in the inverse-Compton cross section. HAWC will measure the spectra of Galactic sources up to $100 \mathrm{TeV}$ to address the question of whether the source spectra exhibit a cutoff or continue to energies well beyond the reach of inverse-Compton upscattering.

- Galactic diffuse emission is produced when cosmic rays interact with interstellar gas and produce pions, which subsequently decay into gamma rays, and when high-energy electrons interact with gas and radiation fields. The measurement of the diffuse flux can therefore be used to find regions of cosmic-ray acceleration in the Galaxy. HAWC is in an excellent position to measure the diffuse gamma-ray spectrum at energies above $10 \mathrm{TeV}$, spatially resolve the diffuse emission, and extend the measurements to $100 \mathrm{TeV}$.

- Active galactic nuclei were among the first extragalactic sources discovered at $\mathrm{TeV}$ energies. They show extreme variability, with flares of up to 50 times their quiescent flux. Their study can provide information on the acceleration mechanisms that produce $\mathrm{TeV}$ gamma rays. High-energy gamma rays from AGN are produced by the interaction of accelerated charged particles with ambient matter or radiation fields. Current models differ in whether electrons or hadrons are accelerated in the sources. In leptonic models, electrons produce high-energy gamma radiation via inverse-Compton scattering of ambient photons; for example, from synchrotron emission, from the accretion disk, or from the cosmic microwave radiation. Hadronic models assume that protons and nuclei are accelerated in the source and interact with interstellar material in an astrophysical beam dump. 
- AGN can also be used to probe the extragalactic background light (EBL) (see, e.g., [4] for a recent review) and the intergalactic magnetic field (IGMF), which gamma rays must traverse from their sources to us (see, e.g., [5, 6] and references therein). Such measurements of the EBL and the IGMF are of considerable importance for cosmology.

- HAWC is a very sensitive detector for cosmic rays. While the large number of cosmic rays detected in HAWC forms an undesirable background in the search for gamma-ray sources, it also enables us to use HAWC for precision measurements of small deviations from isotropy in the cosmic-ray flux. Measurements of cosmic-ray anisotropy at the $10^{-4}$ to $10^{-5}$ level in relative intensity are possible in the next few years.

Examples of other HAWC science topics include tests of Lorentz invariance with transient sources, searches for exotic signals such as massive relic particles (SUSY Q-Balls, WIMP dark matter, Kaluza-Klein dark matter), and primordial black holes. For a recent review of dark matter searches with gamma-ray telescopes, see, e.g., [7].

\section{First Results}

First results from early data include the detection of the cosmic-ray Moon shadow and smallscale anisotropy in the arrival direction distribution of cosmic rays.

The Moon produces a deficit in the arrival direction distribution of cosmic-ray showers reaching the Earth. The observation of this deficit allows for a study of the angular resolution and the pointing accuracy of the detector. Fig. 3 (left) shows a sky map of the region around the Moon for data taken between January and April 2013 with the 30-tank configuration of HAWC. The Moon shadow is observed at high significance. Because of deflection by the Earth's magnetic field, we expect an energy-dependent offset of the Moon shadow from the true position. This offset is mostly in right ascension and amounts to about $1.6^{\circ} \cdot Z$ per energy in $\mathrm{TeV}$, where $Z$ is the charge of the cosmic-ray particle. The expected offset for HAWC has been simulated using models of the Earth's magnetic field, and the observed shift agrees well with the simulation.

Another early result from HAWC data is the detection of anisotropy in the arrival direction distribution of cosmic rays. Anisotropy in the flux of charged particles at $\mathrm{TeV}$ energies is not what one would readily expect. Galactic magnetic fields should scramble the arrival direction distribution of charged primaries in this energy range. Nevertheless, over the last decade, several experiments in the Northern and Southern Hemisphere have reported anisotropy in the arrival direction distribution of cosmic rays at $\mathrm{TeV}$ energies. The observed anisotropy has two main features: a large-scale structure with an amplitude of about $10^{-3}$ usually described as a dipole or a sum of low-order multipoles $[8,9,10,11,12,13]$, and a small-scale structure with a few cosmic-ray excesses and deficits of angular size $10^{\circ}$ to $30^{\circ}[14,15]$. The discovery of this anisotropy clearly implies that the propagation of cosmic rays from their sources to us is not understood.

After several months of operation, the small-scale structure in the cosmic-ray flux is already visible in HAWC data. Fig. 3 (right) shows an equatorial map of the relative intensity of the cosmicray flux as observed by HAWC. The map contains 17.5 billion events taken over 52 days of livetime between June and August 2013 when the detector operated in the 95-tank configuration. This residual map is created by comparing the actual data map to a reference map showing the response 

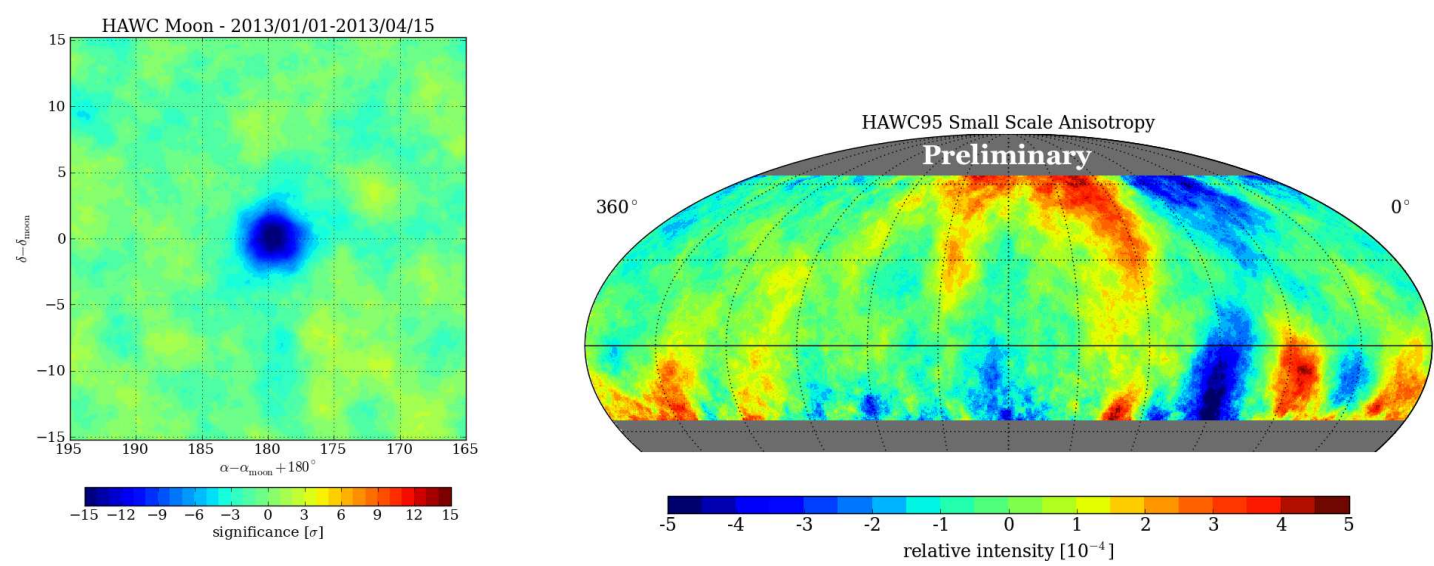

Figure 3: Left: Sky map of the region around the Moon for the 30-tank configuration of HAWC for data taken between January and April 2013. Right: Relative intensity in equatorial coordinates of the arrival direction distribution of cosmic rays as observed in early HAWC data.

of the detector to an isotropic flux of cosmic rays. The reference map itself is not isotropic, as it accounts for changes in the cosmic-ray rate from atmospheric effects, occasional downtime of the detector during construction, and effects from the detector geometry. The techniques used to create the reference map are described in detail in [14]. The maps are produced using the HEALPix library [16] with a resolution of about $1^{\circ}$, but the final map shown in Fig. 3 (right) is smoothed with a $10^{\circ}$ top-hat function to increase the sensitivity to features at that scale.

Several prominent regions of enhanced and reduced cosmic-ray flux are visible in the sky map, and the results are largely in agreement with previous measurements. Milagro's "Region A" and "Region B" [14] appear at high significance. The "Region A" excess around right ascension 54.1 and declination $-8.4^{\circ}$ is measured with a pretrial significance of $7.3 \sigma$, and the "Region B" excess peaks at right ascension $122.3^{\circ}$ and declination $31.0^{\circ}$ with a pretrial significance of $6.5 \sigma$.

The high rate of cosmic ray events detected by HAWC will soon allow us to measure the cosmic-ray anisotropy with unprecedented precision. In addition, HAWC data will be used to study the energy dependence of the anisotropy and to determine whether the spectrum in the region around the "hot spots" is harder or softer than the general cosmic-ray spectrum.

\section{Summary}

Construction of the HAWC observatory is expected to be completed in 2014. Data-taking with the partial detector has already started, and first results already illustrate the dramatic increase in sensitivity over previous TeV all-sky monitors. Within a few months of data-taking, HAWC has observed the cosmic-ray shadow of the Moon and the anisotropy in the arrival direction of cosmic rays. In addition, first results on gamma-ray sources will soon be published.

This work has been supported by the National Science Foundation (NSF-PHY-1306465), the University of Wisconsin Alumni Research Foundation, and the Institute of Geophysics and Plane- 
tary Physics at Los Alamos National Lab.

\section{References}

[1] R. Atkins et al. (Milagro Collaboration), Observation of TeV Gamma Rays from the Crab Nebula with Milagro Using a New Background Rejection Technique, Astrophys. J. 595 (2003) 803 [astro-ph/0305308].

[2] A.U. Abeysekara et al. (HAWC Collaboration), Sensitivity of the High-Altitude Water Cherenkov Detector to Sources of Multi-TeV Gamma Rays, Astropart. Phys. 50-52 (2013) 26 [arXiv:1306.5800].

[3] A.U. Abeysekara et al. (HAWC Collaboration), On the Sensitivity of the HAWC Observatory to Gamma-Ray Bursts, Astropart. Phys. 35 (2012) 641 [arXiv: 1108.6034 ].

[4] E. Dwek and F. Krennrich, The Extragalactic Background Light and the Gamma-Ray Opacity of the Universe, Astropart. Phys. 43 (2013) 112 [arXiv:1209.4661].

[5] K. Dolag, M. Kachelriess, S. Ostapchenko, and R. Tomas, Blazar Halos as Probe for Extragalactic Magnetic Fields and Maximal Acceleration Energy, Astrophys. J. 703 (2009) 1078 [arXiv:0903.2842].

[6] A. Neronov and D.V. Semikoz, Sensitivity of Gamma-Ray Telescopes for Detection of Magnetic Fields in the Intergalactic Medium, Phys. Rev. D 80 (2009) 123012 [arXiv: 0910.1920].

[7] T.A. Porter, R.P. Johnson, and P.W. Graham, Dark Matter Searches with Astroparticle Data, Ann. Rev. Astron. Astrophys. 49 (2011) 155 [arXiv: 1104.2836$].$

[8] M. Amenomori et al. (Tibet AS $\gamma$ Collaboration), Large-Scale Sidereal Anisotropy of Galactic Cosmic-Ray Intensity Observed by the Tibet Air Shower Array, Astrophys. J. 626 (2005) L29 [arXiv:astro-ph/0505114].

[9] K. Munakata et al.(Kamiokande Collaboration), Large-Scale Anisotropy of the Cosmic-Ray Muon Flux in Kamiokande, Phys. Rev. D 56 (1997) 23.

[10] G. Guillian et al. (Super-Kamiokande Collaboration), Observation of the Anisotropy of 10-TeV Primary Cosmic-Ray Nuclei Flux with the Super-Kamiokande-I Detector, Phys. Rev. D 75 92007) 062003 [arXiv: astro-ph/0508468].

[11] A.A. Abdo et al. (Milagro Collaboration), The Large-Scale Cosmic-Ray Anisotropy as Observed with Milagro, Astrophys. J. 69821212009 [arXiv: 0806.2293].

[12] M. Aglietta et al. (EAS-TOP Collaboration), Evolution of the Cosmic-Ray Anisotropy Above $10^{14} \mathrm{eV}$, Astrophys. J. 692 (2009) L130 [arXiv: 0901.2740 ].

[13] R.U. Abbasi et al. (IceCube Collaboration), Measurement of the Anisotropy of Cosmic-Ray Arrival Directions with IceCube, Astrophys. J. 718 (2010) L194 [arXiv: 1005 .2960].

[14] A.A. Abdo et al.(Milagro Collaboration), Discovery of Localized Regions of Excess 10-TeV Cosmic Rays, Phys. Rev. Lett. 101 (2008) 221101 [arXiv: 0801.3827 ].

[15] R.U. Abbasi et al. (IceCube Collaboration), Observation of Anisotropy in the Arrival Directions of Galactic Cosmic Rays at Multiple Angular Scales with IceCube, Astrophys. J. 740 (2011) 16 [ (arXiv:1105.2326].

[16] K.M. Gorski et al., HEALPix - a Framework for High Resolution Discretization and Fast Analysis of Data Distributed on the Sphere, Astrophys. J. 622 (2005) 759 [astro-ph/ 0409513 ]. 